\title{
Correlação entre estado nutricional e força de preensão palmar em idosos
}

\section{Corredionbedveenntitiond stusandhandgipstrengthinedaly}

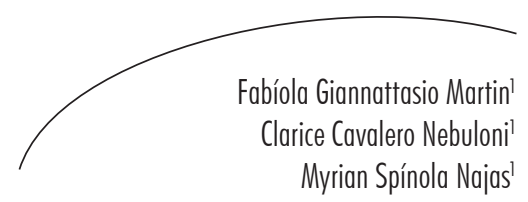

\section{Resumo}

No envelhecimento ocorrem mudanças corpóreas, dentre as quais a diminuição de massa magra com prejuízo na força muscular, afetando a capacidade funcional. Atualmente, o teste de Força de Preensão Palmar (FPP) está sendo utilizado para avaliar a força muscular global como auxiliar na avaliação nutricional. Objetivo: Verificar a correlação entre o estado nutricional e a FPP em idosos. Métodos: Estudo transversal realizado com idosos atendidos em ambulatórios de uma universidade pública federal. Para a classificação do estado nutricional, utilizam-se: índice de massa corpórea (IMC), avaliação nutricional por meio da Mini-Avaliação Nutricional (MAN), circunferência da panturrilha (CP) e FPP realizada com auxílio de um dinamômetro manual hidráulico. Utilizaram-se como referência para a FPP e IMC os pontos de cortes divididos por gênero (projeto SABE/OPAS). Resultados: Amostra constituída ( $\mathrm{n}=42$ ) na sua maioria por mulheres $(66,7 \%)$, apresentando em média 26,82 pontos na MAN e pelo IMC a maioria dos idosos eram eutróficos - 46,4\% mulheres e 57,1\% homens. Homens e mulheres obtiveram média superior a $31 \mathrm{~cm}$ de circunferência da panturrilha (CP). A média de força para homens foi superior ao valor obtido pelas mulheres. Não houve diferença estatisticamente significante entre o estado nutricional classificado pelo IMC e a FPP para homens e mulheres; houve correlação estatisticamente significante ( $\mathrm{p}$ $0,008)$ entre o estado nutricional classificado pela MAN e a força de preensão palmar na amostra total. Conclusões: Dentre os métodos para avaliar o estado nutricional, apenas a MAN correlacionou-se positivamente com a FPP, que é uma medida associada à funcionalidade em idosos.

\section{Abstract}

In the aging process, body changes occur, such as reduction of lean mass with impaired muscle strength, affecting the functional capacity. Currently, the Hand Grip Strength Test (HGST) is being used to evaluate the overall muscle strength as an aid in nutritional assessment. Objective: To investigate the correlation between the HGST and nutritional

Curso de especialização em Gerontologia. Departamento de Medicina. Universidade Federal de São Paulo. São Paulo, SP, Brasil.

Correspondência / Correspondence

Fabíola Giannattasio Martin

E-mail: bila_martin@yahoo.com.br 
status in the elderly. Methods: Cross sectional study on elderly people attending outpatient of a Federal Public University. To evaluate the nutritional status, were used: body mass index (BMI), nutritional evaluation by Mini Nutritional Assessment (MNA), calf circumference and HGST performed with the aid of a hydraulic hand dynamometer. It was used as reference for the GSTP and the BMI, cutoff points divided by gender (SABE / OPAS Project). Results: The sample was mostly formed $(\mathrm{n}=42)$ by women $(66.7 \%)$, with an average of 26.82 points in the MNA and BMI, most seniors were eutrophic - 46.4\% women and 57.1\% men. Men and women were averaged over 31 $\mathrm{cm}$ of calf circumference. The average strength for men was higher than women. There was no statistically significant difference between nutritional status classified by BMI and HGST for men and women, there was a statistically significant correlation ( $\mathrm{p}$ 0.008) between the nutritional status classified by MNA and hand grip strength in the total sample. Conclusions: Among the methods to assess nutritional status, only MNA correlated positively with the HGST, which is a measure associated with the functionality in the elderly.
Key words: Aging. Nutrition Assessment. Dynamometer Muscle Strength. Muscle strength.

\section{INTRODUÇÃO}

No processo de envelhecimento, ocorrem mudanças corpóreas consideradas normais, em homens e mulheres. Essas alterações incluem uma progressiva diminuição da massa corporal magra e de líquidos corpóreos, aumento da quantidade de tecido gorduroso, declínio do tamanho e peso de vários órgãos (rins, fígado, pulmões) e, sobretudo, uma grande perda de músculos esqueléticos. ${ }^{1}$ Este processo pode ser acelerado por diversos fatores, entre eles a má nutrição, a presença de múltiplas doenças crônicas e a inatividade física, prejudicando a mobilidade e afetando de forma negativa o desempenho das atividades de vida diária. ${ }^{2}$ Concomitantemente a essas alterações, ocorre um decréscimo da força muscular, potencializando as deficiências e limitações funcionais em idosos.

Indivíduos que apresentam redução de massa muscular e, consequentemente, de força, terão prejuízos na execução das atividades diárias. ${ }^{3}$ A redução de massa muscular tem como principal característica a diminuição do músculo esquelético, bem como de seu desempenho, ${ }^{4}$ podendo ocorrer antes do surgimento das manifestações clínicas e do prejuízo funcional, ${ }^{5}$ conferindo aos idosos maior probabilidade de quedas, fraturas, incapacidade, dependência, hospitalizações recorrentes e aumento da mortalidade.
Os homens apresentam perda muscular superior à feminina, porém a perda de força é mais intensa nas mulheres em decorrência da menor proporção de massa muscular e maior expectativa de vida. ${ }^{4} \mathrm{~A}$ perda de massa muscular é também associada à morte de motoneurônios, redução de células propriamente dita, provocadas pela inatividade, como também pela diminuição de hormônios como a testosterona e o hormônio do crescimento.

As habilidades funcionais e a força muscular, além de estarem associadas à massa muscular, estão associadas ao estado nutricional do indivíduo. Segundo a Associação Americana de Saúde Pública, o estado nutricional (EN) é definido como a "condição de saúde de um indivíduo influenciado pelo consumo e utilização de nutrientes e identificada pela correlação de informações obtidas através de estudos físicos, bioquímicos, clínicos e dietéticos". O $\mathrm{EN}$, portanto, é detectado com base em vários parâmetros, que podem ser utilizados e avaliados de forma isolada ou associada. ${ }^{6}$

A avaliação do EN do idoso é um processo complexo, que possibilitaaintervenção nutricional adequada evitando ou minimizando agravos à saúde que comprometam a capacidade funcional. Vários instrumentos foram desenvolvidos para avaliar o estado nutricional de idosos, dentre eles, a Mini-Avaliação Nutricional (MAN). ${ }^{7}$ Trata-se de um método validado e considerado padrão 
ouro ${ }^{8}$ para esta população, por ser prático, não invasivo, de simples mensurações e de questões rápidas, aplicadas em cerca de 10 minutos, ${ }^{9}$ desde que realizado por profissional bem treinado.

O índice de massa corpórea (IMC) é um bom indicador do EN do idoso. É muito utilizado devido à facilidade de aplicação, à existência de padrões de referência, que permitem comparações entre populações, à boa relação com a morbimortalidade e, ainda, por não ser invasivo, de baixo custo e mais preciso que as medidas obtidas por meio das dobras cutâneas. ${ }^{10}$

Outro parâmetro muito utilizado e considerado uma medida antropométrica bastante importante e indicadora de massa muscular é a circunferência da panturrilha (CP), que fornece uma estimativa da reserva proteica e pode ser utilizada em programas de avaliação nutricional, auxiliando na detecção de riscos, de forma a garantir intervenções adequadas, melhorando a qualidade de vida do idoso. ${ }^{11}$

Atualmente, outro método simples que vem sendo utilizado para o acompanhamento do EN é a dinamometria manual, ${ }^{12}$ mais conhecido como Teste de Força de Preensão Palmar. Consiste na aferição da força máxima voluntária de preensão manual e tem como princípio estimar a função do músculo esquelético. ${ }^{13} \mathrm{O}$ dinamômetro é um aparelho de simples manuseio, que fornece leitura rápida e direta, além de permitir fácil utilização tanto em estudos de campo quanto em situações clínicas ambulatoriais. ${ }^{14}$

A aplicação do teste de FPP urge como uma alternativa simples, objetiva, de baixo custo e pouco invasiva. Contudo, o maior obstáculo para ser utilizado como um instrumento de avaliação nutricional consiste no fato de que ainda não há um padrão de referência para a população idosa. ${ }^{14}$ É um teste utilizado para screening, para indicar riscos à saúde, relacionados à força muscular, ${ }^{2,15}$ pois a avaliação da FPP tem sido correlacionada com a força muscular dos demais músculos do organismo; é considerado um bom indicador para a força muscular global, bem como para avaliar o desempenho físico dos idosos.
A força de preensão torna-se relevante indicador de alterações do EN em curto prazo, bem como da resposta ao suporte nutricional, descrito como um dos mais sensíveis testes funcionais indicadores de depleção proteica. ${ }^{14} \mathrm{~A}$ baixa funcionalidade pode ser mensurada pelo teste da FPP e associa-se ao EN insatisfatório, independentemente da idade, sexo ou estatura. ${ }^{16}$ Por conseguinte, a avaliação da força muscular pode auxiliar em condutas de intervenção para prevenir ou retardar futuras limitações e deficiências funcionais. ${ }^{15}$

O conhecimento da correlação entre o estado nutricional e a força de preensão palmar como preditor de funcionalidade poderá direcionar novas estratégias de promoção, prevenção e tratamento de agravos à saúde em idosos, visando à melhoria da sua qualidade de vida. $\mathrm{O}$ objetivo deste artigo, foi verificar a correlação entre o estado nutricional e força de preensão palmar em idosos atendidos em assistência ambulatorial.

\section{METODOLOGIA}

Estudo transversal realizado com idosos atendidos nos ambulatórios da disciplina de Geriatria e Gerontologia da Universidade Federal de São Paulo (UNIFESP). Os dados foram coletados no período de julho a outubro de 2009. A amostra constituiu-se de idosos do gênero masculino e feminino, com idade $\geq 60$ anos, atendidos em primeira consulta e que não apresentassem critérios de exclusão. Para a coleta de dados, aplicou-se a anamnese com questões para determinar o perfil da amostra, contendo dados pessoais, comorbidades, medicamentos em uso e prática de exercício físico. ${ }^{17}$ Para a avaliação nutricional, foram coletados dados antropométricos e aplicados a $\mathrm{MAN}^{7}$ e o teste de FPP.

Foram excluídos os idosos com osteoartrite/ osteoartrose de mãos, ombros, e difusa; presença de fatores de risco cardiovasculares não controlados; diagnóstico ortopédico ou neurológico que inviabilizasse e alterasse a execução dos testes, como amputação, ombro doloroso, assimetrias, doença de Parkinson, 
alteração de tônus e doença cerebelar ou déficit cognitivo que não permitisse a compreensão dos comandos.

\section{Avaliação nutricional}

A avaliação nutricional foi composta por: avaliação antropométrica, aplicação do FPP e MAN. Para a avaliação antropométrica, foram avaliadas as medidas de peso (aferido em pé, descalços, com o mínimo de vestuário, utilizando-se balança Filizola, capacidade de 150 $\mathrm{kg}$ e sensibilidade de 100g); estatura (medida durante a expiração, utilizando o antropômetro da balança com o idoso em pé, sem sapatos, com a cabeça sem a utilização de adornos e formando um ângulo de $90^{\circ}$ entre o queixo e pescoço); circunferência de braço - CB (aferida no ponto médio do braço esquerdo entre o acrômio e o olécrano, sendo esta medida coletada para ser registrada na MAN; e circunferência de panturrilha - CP (aferida no maior volume da perna esquerda com o idoso sentado em uma cadeira com a perna flexionada a $90^{\circ}$ ). As medidas de circunferência foram realizadas com o auxílio de fita métrica inelástica, com precisão de $1 \mathrm{~mm}$. A unidade de medida adotada foi o centímetro.

A força de preensão palmar foi realizada com o auxílio de um dinamômetro manual hidráulico da marca Saehan (Saehan corporation - SH5001), adotando a unidade de medida em quilogramas $(\mathrm{kg})$. A escala vai progredindo de dois em dois quilos, iniciando-se em quatro quilos. $\mathrm{O}$ idoso permaneceu sentado em cadeira com o braço aduzido e em rotação neutra, com cotovelo flexionado a $90^{\circ}$, com antebraço e punho em rotação neutra. A empunhadura foi autoajustada, conforme o relato de maior conforto dado pelo paciente e após a observação da posição correta do aparelho, cuja haste foi posicionada entre as segundas falanges dos dedos (indicador, médio e anular). ${ }^{18}$ No início do teste, a agulha foi colocada na posição neutra (zero). Ao comando de voz do avaliador, o idoso deveria realizar o máximo de força para aproximar as duas hastes do aparelho. Coletaram-se três medidas de ambos os braços num intervalo de descanso de um minuto entre elas, utilizando-se o maior valor obtido. ${ }^{19}$ A metodologia de aferição foi baseada nas instruções descritas no manual do fabricante.

\section{Classificação do estado nutricional}

Para a classificação do EN, utilizaramse os dados de: 1) índice de massa corpórea (IMC), que é uma medida secundária obtida por meio de duas medidas primárias: peso $(\mathrm{kg})$ dividido pelo quadrado da estatura (m); 2) teste de Força de Preensão Palmar (FPP); 3) MiniAvaliação Nutricional (MAN); e 4) medida de circunferência da panturrilha.

\section{Padrões de referências adotados para} a classificação do estado nutricional

IMC: os valores de referência de IMC adotados foram do projeto $\mathrm{SABE} / \mathrm{OPAS}^{2}$, que utiliza como pontos de corte para normalidade ou eutrofia, o IMC entre $23 \mathrm{~kg} / \mathrm{m}^{2}$ e $28 \mathrm{~kg} / \mathrm{m}^{2}$; desnutridos, valores abaixo do IMC $23 \mathrm{~kg} / \mathrm{m}^{2}$; sobrepeso, IMC entre 28 e $30 \mathrm{~kg} / \mathrm{m}^{2}$; e obesos aqueles com valores acima de $30 \mathrm{~kg} / \mathrm{m}^{2}$.

MAN: escore adequado, $\geq 24$ pontos; risco de desnutrição, entre 17 e 23,5 pontos; desnutrição $<17$ pontos.

FPP: dados publicados pelo projeto SABE/ OPAS $^{2}$, divididos por gênero e pelo IMC.

CP: foi considerada adequada a circunferência igual ou superior a $31 \mathrm{~cm}$ para homens e para mulheres. ${ }^{7}$

Para a análise dos dados, empregaram-se os seguintes softwares: SPSS V16, Minitab 15 e Excel Office 2007. Aplicou-se a análise de correlação de Spearman para medir o grau de associação entre duas variáveis, distribuição $t$ de Student com (n-2) graus de liberdade; o Teste de Mann-Whitney e o Teste de Igualdade de duas 
Proporções. O intervalo de confiança para a média adotado foi de $95 \%$. Em todos os testes, fixou-se em 0,05 ou $5 \%$ o nível de rejeição da hipótese de nulidade, assinalando com asterisco os valores significantes. Os idosos participantes do estudo assinaram o termo de consentimento livre e esclarecido (aprovado pelo Comitê de Ética no 1010/09), cumprindo os princípios éticos na Declaração de Helsinki, além do atendimento à legislação pertinente.

\section{RESULTADOS}

A amostra constituiu-se de 42 indivíduos. As mulheres representaram $66,7 \%$ da amostra $(n=28)$ e os homens, 33,3\% ( $n=14)$, sendo esta diferença entre os gêneros significativa $(p=$ $0,002)$. A etnia predominante foi a branca, com $61,9 \%$ dos idosos, seguida por $19 \%$ da amarela e $9,5 \%$ da negra e parda.

A idade média dos idosos foi de 74,07 anos. A média do gênero feminino foi de 72,39 anos, variando de 64 a 86 anos. Para o gênero masculino, a média de idade foi de 77,43 anos, variando de 64 a 87 anos. A média da escolaridade foi de 6,38 anos, predominando um a quatro anos de escolaridade, tanto para homens quanto para mulheres, sendo os analfabetos a minoria (7,14\%). A média de comorbidades apresentadas para homens e mulheres foi de 6,17, variando de duas doenças no mínimo e 13 no máximo. Metade da amostra apresentou mais de seis comorbidades.

Cinquenta por cento dos idosos utilizavam mais de três medicamentos, sendo que o número máximo utilizado foi de 15 . O consumo diário de cinco ou mais medicamentos (polifarmácia) ${ }^{20}$ foi verificado em $21,4 \%$ da amostra total.

A média da MAN foi de 26,82 pontos - 26,5 pontos para o gênero feminino e 27,46 para o masculino. Apenas dois indivíduos, tanto na amostra feminina quanto na masculina, apresentaram risco nutricional pela MAN, representando $7,1 \%$ nas mulheres e $14,3 \%$ nos homens.

A seguir, na tabela 1 , a descrição da amostra com os dados citados anteriormente (exceto etnias).

Tabela 1 - Análise descritiva das variáveis idade (anos), escolaridade (anos), valores obtidos pela MAN, número de comorbidades e número de medicamentos de idosos do gênero masculino e feminino. São Paulo, SP, 2009.

\begin{tabular}{lcccccc}
\hline Descritiva & Média & Mediana & $\begin{array}{c}\text { Desvio } \\
\text { Padrão }\end{array}$ & Min & Max & IC \\
\hline Idade & 74,07 & 75,5 & 6,24 & 64,0 & 87,0 & 1,89 \\
Escolaridade & 6,38 & 4,0 & 4,08 & 0,0 & 12,0 & 1,24 \\
MAN & 26,82 & 27,5 & 2,47 & 17,5 & 30,0 & 0,75 \\
N comorb & 6,17 & 6,0 & 2,70 & 2,0 & 13,0 & 0,82 \\
N medic & 3,69 & 3,0 & 3,14 & 0,0 & 15,0 & 0,95 \\
\hline
\end{tabular}

A prática de exercício físico (EF) observada nos idosos foi de 64,3\% e 78,6\%, em mulheres e homens, respectivamente, enquanto que
$35,7 \%$ das mulheres e $21,4 \%$ dos homens eram sedentários, classificados pelo IPAQ. ${ }^{17}$ 
Tabela 2 - Análise descritiva das variáveis: IMC $\left(\mathrm{kg} / \mathrm{m}^{2}\right)$, FPPD e FPPE $(\mathrm{kg}), \mathrm{CB}(\mathrm{cm})$ e CP $(\mathrm{cm})$ e MAN de idosos do gênero masculino e feminino. São Paulo, SP, 2009.

\begin{tabular}{|c|c|c|c|c|c|c|c|c|c|c|c|c|c|}
\hline \multirow[b]{2}{*}{ Variáveis } & \multicolumn{6}{|c|}{ HOMENS $(\mathrm{n}=14)$} & \multicolumn{6}{|c|}{ MULHERES $(\mathrm{n}=28)$} & \multirow[b]{2}{*}{$\mathrm{P}$ valor } \\
\hline & Média & Mediana & $\mathrm{DP}$ & Mín & Máx & IC & Média & Mediana & $\mathrm{DP}$ & Mín & Máx & IC & \\
\hline $\operatorname{IMC}\left(\mathrm{kg} / \mathrm{m}^{2}\right)$ & 26,23 & 27,1 & 3,77 & 18,70 & 32,15 & 1,98 & 26,35 & 27,1 & 3,82 & 19,16 & 33,26 & 1,42 & 0,873 \\
\hline FPPD (kg) & 35 & 33 & 7,22 & 26 & 48 & 3,78 & 20,61 & 21 & 5,14 & 12 & 31 & 1,91 & $<0,001 *$ \\
\hline FPPE (kg) & 32,29 & 32 & 6,22 & 22 & 38 & 3,26 & 19,43 & 20 & 4,07 & 12 & 26 & 1,51 & $<0,001 *$ \\
\hline $\mathrm{CB}(\mathrm{cm})$ & 29,44 & 29,4 & 3,52 & 23,5 & 34,8 & 1,47 & 29,62 & 30,1 & 3,53 & 23,6 & 37,3 & 1,47 & 0,858 \\
\hline $\mathrm{CP}(\mathrm{cm})$ & 35,42 & 35,4 & 2,57 & 31,1 & 39 & 1,40 & 35,89 & 36,3 & 2,98 & 29 & 41,6 & 1,25 & 0,561 \\
\hline MAN & 27,46 & 28,5 & 2,44 & 22,5 & 29,5 & 1,28 & 26,50 & 26,8 & 2,47 & 17,5 & 30 & 0,92 & 0,073 \\
\hline
\end{tabular}

IMC: índice de massa corpórea; FPPD: teste de força de preensão palmar realizado com a mão direita; FPPE: teste de força de preensão palmar realizado com a mão esquerda; CB: circunferência do braço; CP: circunferência da panturrilha; MAN: Mini-Avaliação Nutricional.

Conforme se observa na tabela 2 , a média de IMC encontrada nas mulheres foi de 26,35 $\mathrm{kg} / \mathrm{m}^{2}$, e nos homens, $26,23 \mathrm{~kg} / \mathrm{m}^{2}$, diferença não estatisticamente significante. Os homens apresentaram maior força de preensão palmar, se comparados às mulheres, tanto para a mão direita quanto para a esquerda. Estes dados foram estatisticamente significativos. Não houve diferença estatisticamente significante na média de circunferência de panturrilha entre homens e mulheres.
Segundo o IMC na amostra feminina, $46,4 \%$ apresentavam diagnóstico nutricional de eutrofia, seguidos por 21,4\% com diagnóstico de desnutrição e sobrepeso e 10,8\% com obesidade. Dentre os idosos, mais da metade da amostra $(57,1 \%)$ obteve diagnóstico nutricional de eutrofia, seguidos por $21,5 \%$ com sobrepeso, $14,3 \%$ com diagnóstico de desnutrição e somente $7,1 \%$ da amostra apresentando obesidade.

As tabelas 3 e 4 descrevem os valores de força de preensão palmar segundo as faixas de IMC para mulheres e homens, respectivamente.

Tabela 3 - Análise descritiva do teste de força de preensão palmar de mulheres, segundo faixa de IMC $\left(\mathrm{kg} / \mathrm{m}^{2}\right)$. São Paulo, SP, 2009.

\begin{tabular}{lcccc|cccc}
\hline \multirow{2}{*}{ Feminino } & \multicolumn{9}{c}{ FPPD } \\
\cline { 2 - 9 } Média & Até 23 & De 23 a 28 & De 28 a 30 & Mais 30 & Até 23 & De 23 a 28 & De 28 a 30 & Mais 30 \\
Mediana & 22,00 & 18,46 & 25,67 & 21,33 & 20,00 & 18,15 & 22,67 & 20,00 \\
Desvio Padrão & 22,0 & 20,0 & 28,0 & 23,0 & 20,0 & 18,0 & 26,0 & 21,0 \\
Q1 & 2,19 & 4,98 & 6,81 & 5,47 & 2,19 & 3,95 & 5,77 & 4,73 \\
Q3 & 20,5 & 14,0 & 23,0 & 19,0 & 18,0 & 16,0 & 21,0 & 18,5 \\
N & 22,0 & 22,0 & 29,5 & 25,5 & 22,0 & 22,0 & 26,0 & 22,0 \\
IC & 6 & 13 & 3 & 6 & 6 & 13 & 3 & 6 \\
p-valor & 1,75 & 2,71 & 7,70 & 4,37 & 1,75 & 2,15 & 6,53 & 3,79 \\
\hline
\end{tabular}


Tabela 4 - Análise descritiva do teste de Força de Preensão Palmar de homens, segundo faixa de IMC. São Paulo, SP, 2009.

\begin{tabular}{lcccc|cccc}
\hline \multirow{2}{*}{ Masculino } & \multicolumn{9}{c}{ FPPD } \\
\cline { 2 - 9 } Média & Até 23 & De 23 a 28 & De 28 a 30 & Mais 30 & Até 23 & De 23 a 28 & De 28 a 30 & Mais 30 \\
Mediana & 27,00 & 36,75 & 34,00 & 40,00 & 26,00 & 33,25 & 30,67 & 42,00 \\
Desvio Padrão & 27,0 & 37,0 & 30,0 & 40,0 & 26,0 & 33,0 & 32,0 & 42,0 \\
Q1 & 1,41 & 7,01 & 8,72 & - x - & 2,83 & 6,23 & 4,16 & - x - \\
Q3 & 26,5 & 32,0 & 29,0 & 40,0 & 25,0 & 31,0 & 29,0 & 42,0 \\
N & 27,5 & 40,5 & 37,0 & 40,0 & 27,0 & 38,5 & 33,0 & 42,0 \\
IC & 2 & 8 & 3 & 1 & 2 & 8 & 3 & 1 \\
p-valor & 1,96 & 4,85 & 9,86 & $-x-$ & 3,92 & 4,32 & 4,71 & - x - \\
\hline
\end{tabular}

As mulheres apresentaram valores de força de preensão palmar inferiores à dos homens em todas as faixas de IMC. Idosas desnutridas apresentaram força de preensão palmar semelhante à das idosas obesas, tanto para mão direita quanto para a mão esquerda (tabela 3). Já os homens desnutridos apresentaram força de preensão palmar inferiores à dos idosos obesos em ambas as mãos (tabela 4).
Observa-se que não existiram diferenças estatisticamente significativas nas faixas de IMC para os valores do teste de FPP realizado com a mão direita (FPPD) e do teste de FPP realizado com a mão esquerda (FPPE) entre homens e mulheres. Pode-se observar, na tabela 5 , que não houve diferença estatística nos valores do teste de força de preensão palmar entre os idosos que praticavam exercício físico e os sedentários.

Tabela 5 - Associação entre FPP e Exercício Físico. São Paulo, SP, 2009.

\begin{tabular}{|c|c|c|c|c|}
\hline \multirow{2}{*}{ Exercício Físico } & \multicolumn{2}{|c|}{ FPPD } & \multicolumn{2}{|c|}{ FPPE } \\
\hline & Não & $\operatorname{Sim}$ & Não & $\operatorname{Sim}$ \\
\hline Média & 26,86 & 22,15 & 24,62 & 21,69 \\
\hline Mediana & 26,0 & 22,0 & 22,0 & 22,0 \\
\hline Desvio Padrão & 8,89 & 8,74 & 7,98 & 7,25 \\
\hline Q1 & 20,0 & 18,0 & 20,0 & 16,0 \\
\hline Q3 & 31,0 & 26,0 & 32,0 & 26,0 \\
\hline $\mathrm{N}$ & 29 & 13 & 29 & 13 \\
\hline IC & 3,23 & 4,75 & 2,91 & 3,94 \\
\hline p-valor & \multicolumn{2}{|c|}{0,123} & \multicolumn{2}{|c|}{0,233} \\
\hline
\end{tabular}

Conforme se observa na tabela 6 , houve correlação estatisticamente significante $(p=$ $0,008 ; \mathrm{p}=0,002)$ entre a MAN e a força de preensão palmar na amostra total. Essa correlação se mantém significativa para FPPE somente em relação à amostra feminina $(\mathrm{p}=$ $0,029)$ - tabelas 6,7 e 8. 
Tabela 6 - Correlação entre a MAN, circunferência da panturrilha e FPP na amostra total. São Paulo, SP, 2009.

\begin{tabular}{|c|c|c|c|}
\hline \multicolumn{2}{|c|}{ Geral } & \multirow{2}{*}{$\frac{\text { FPPD }}{40,4 \%}$} & \multirow{2}{*}{$\begin{array}{l}\text { FPPE } \\
45,7 \%\end{array}$} \\
\hline MAN & corr & & \\
\hline & p-valor & $0,008^{*}$ & $0,002 *$ \\
\hline \multirow[t]{2}{*}{$\mathrm{CP}$} & corr & $14,1 \%$ & $10,7 \%$ \\
\hline & p-valor & 0,418 & 0,543 \\
\hline
\end{tabular}

Tabela 7 - Correlação entre a MAN, circunferência da panturrilha e FPP na amostra feminina. São Paulo, SP, 2009.

\begin{tabular}{|c|c|c|c|}
\hline \multicolumn{2}{|c|}{ Feminino } & \multirow{2}{*}{$\begin{array}{l}\text { FPPD } \\
30,2 \%\end{array}$} & \multirow{2}{*}{$\begin{array}{l}\text { FPPE } \\
41,2 \%\end{array}$} \\
\hline MAN & corr & & \\
\hline & p-valor & 0,118 & \\
\hline \multirow[t]{2}{*}{$\mathrm{CP}$} & corr & $30,9 \%$ & $28,4 \%$ \\
\hline & p-valor & 0,162 & 0,201 \\
\hline
\end{tabular}

Tabela 8 - Correlação entre a MAN, circunferência da panturrilha e FPP na amostra masculina. São Paulo, SP, 2009.

\begin{tabular}{|c|c|c|c|}
\hline \multicolumn{2}{|c|}{ Masculino } & \multirow{2}{*}{$\begin{array}{l}\text { FPPD } \\
10,8 \%\end{array}$} & \multirow{2}{*}{$\begin{array}{l}\text { FPPE } \\
19,5 \%\end{array}$} \\
\hline TMIS & corr & & \\
\hline MULV & p-valor & 0,714 & 0,504 \\
\hline \multirow{2}{*}{$\mathrm{CP}$} & corr & $41,4 \%$ & $35,7 \%$ \\
\hline & p-valor & 0,159 & 0,231 \\
\hline
\end{tabular}

Ao considerarmos o estado nutricional pelo parâmetro da circunferência da panturrilha, observamos que não houve diferença estatisticamente significante entre FPP e CP (tabelas 6, 7 e 8).

\section{DISCUSSÃO}

No presente estudo, observou-se maior proporção de mulheres $(66,7 \%)$ em relação aos homens, sugerindo maior participação das mulheres nos serviços de saúde, inclusive nos de geriatria. ${ }^{21}$
Houve predomínio da etnia branca no estudo, o que pode ser explicado pela ausência de mudanças étnicas da população idosa brasileira como um todo até o ano de 2000. Dos 14,5 milhões de idosos, $60,7 \%$ eram brancos $7,0 \%$ negros e 30,7\% pardos. Os amarelos e indígenas constituem uma parcela pequena da população idosa, $1,2 \% .^{21}$

A força muscular tende a ser diferente entre os gêneros, sendo que as mulheres apresentam valores inferiores aos dos homens. Quando comparada à idade, porém, a força sofre um declínio com o envelhecimento. ${ }^{22}$ Existe forte 
associação inversa entre a força de preensão palmar e a idade. ${ }^{23}$

Alexandre ${ }^{2}$ em estudo avaliando força de preensão palmar e sua relação com capacidade funcional, também obteve resultados semelhantes, tendo os homens apresentado maior força de preensão se comparados às mulheres. Isto se explica pelo fato de os homens possuírem mais massa magra por características fisiológicas, justificadas pela maior concentração de testosterona, hormônio do crescimento $(\mathrm{GH})$, insulina, que contribui para o aumento do hormônio do crescimento 1 (IGF-1), e dehidropiandrosterona (DHEA) que aumenta o turnover protéico muscular. ${ }^{2}$

Moura $^{22}$ encontrou como média de força entre os idosos (homens e mulheres): $28,11 \mathrm{~kg}$ para FPPD e 25,73kg para FPPE. A média obtida pelos idosos do gênero masculino foi $35,69 \mathrm{~kg}$ na mão direita e $32,47 \mathrm{~kg}$ na mão esquerda. A média feminina foi de $20,55 \mathrm{~kg}$ na mão direita e de $19,03 \mathrm{~kg}$ na mão esquerda. Ao compararmos os resultados desse pesquisador, podemos verificar grande semelhança com os resultados obtidos no presente estudo. Contudo, independentemente do perfil do idoso, estudos sugerem que valores inferiores a $20 \mathrm{~kg}$ representam risco para dependência futura e baixos níveis de saúde. ${ }^{24}$

A grande discrepância entre a força muscular de homens e mulheres da amostra é uma tendência fisiológica bem conhecida, relatada também nos demais estudos. Tanto os homens quanto as mulheres seguem o mesmo padrão de força durante a vida e mesmo durante o envelhecimento, e a perda se mantém proporcional. $^{22}$

Também é citada na literatura que a força de preensão palmar está correlacionada à força física, força cognitiva e de comorbidades relacionadas à idade, e que os homens têm valores médios maiores de FPP em todas as fases da vida, comparados às mulheres. ${ }^{22}$ Quando os valores de força de preensão palmar são ajustados à idade e ao gênero, os valores estão mais associados à estatura, massa muscular e área muscular do braço (AMB) do que ao IMC e à porcentagem de massa gorda. ${ }^{23}$

A FPP é utilizada para avaliar a evolução do paciente. $\mathrm{O}$ pico da força manual ocorre entre os 20 e 40 anos de idade, ocorrendo seu declínio com o avançar da idade (principalmente a força isométrica). Tal declínio é atribuído aos fatores antropométricos como diminuição do peso, estatura, AMB e densidade óssea, diminuição da atividade física, redução dos músculos das mãos, declínio da eficiência muscular e alteração da composição corporal. ${ }^{2,25}$

O termo "dinapenia" é também utilizado para definir a perda de força muscular relacionada ao envelhecimento, sendo reflexo de alterações na prática de atividade física, fatores neurológicos e musculares, dissociando a perda de massa e força muscular. ${ }^{26}$

Segundo a coorte de Rantanen ${ }^{15}$ que avaliou homens com idade média de 54 anos inicialmente saudáveis, foi possível predizer deficiências e limitações por meio da medida da força de preensão palmar 25 anos depois. Indivíduos que mantêm valores altos da medida da FPP na meia idade têm menores chances de se tornarem incapazes por terem maiores reservas de força que os livra de desenvolver condições crônicas de incapacidade funcional. ${ }^{15}$

No estudo de Giampaoli ${ }^{1}$ em que a amostra era dividida em idosos sem incapacidades e incapazes, de acordocom a capacidade derealizar as Atividades de Vida Diária (AVDs) e Atividades Instrumentais de Vida Diária (AIVDs), foi possível verificar a relação positiva entre o resultado do teste de FPP e a severidade da incapacidade. Quanto menor o valor do teste de FPP maior eram as deficiências/ incapacidades dos indivíduos. Fica claro perceber que, quanto menor a força empregada no teste de preensão palmar maiores são os riscos para limitações futuras. ${ }^{15}$

Alexandre $^{2}$ verificou que os valores da força de preensão palmar diminuem de acordo 
com a queda do valor do IMC. No presente estudo, os homens desnutridos apresentaram força de preensão palmar inferior à dos idosos obesos, o que corrobora esse estudo. Já as idosas desnutridas apresentaram força de preensão palmar semelhante à das idosas obesas. ${ }^{2}$

Ao avaliarmos a importância do IMC sobre a força muscular, percebemos que esta força é afetada pelo estado nutricional logo, é esperado que indivíduos com pior diagnóstico nutricional não apresentem valores de força muscular superiores aos indivíduos mais bem nutridos. Embora indivíduos com valores baixos de IMC tenham maiores chances de apresentar valores reduzidos de força de preensão palmar, comparados aos indivíduos com valores normais de IMC 15, não houve diferença estatisticamente significativa. Entretanto, quando utilizamos a Mini-Avaliação Nutricional, que considera outros parâmetros além dos antropométricos, verificamos correlação positiva entre o escore da MAN e o teste de FPP para a amostra total, ou seja, quanto maior o escore apresentado na Mini-Avaliação Nutricional, maior a força de preensão palmar.

A CP apresenta forte associação com a reserva de massa muscular e tem sido recomendada como medida sensível da perda de massa muscular em idosos, especialmente se a redução se dá em função da diminuição da atividade física. ${ }^{3}$ Apesar de homens e mulheres terem apresentado valor médio de circunferência da panturrilha acima de $31 \mathrm{~cm}$, não houve diferença significante entre a medida de CP e FPP.

A atividade física está diretamente relacionada à força muscular. Idosos sedentários apresentam déficits de massa corporal, bem como baixos valores de FPP e, consequentemente, maiores limitações funcionais. Logo, o teste de FPP é utilizado como parâmetro para força muscular global e funcionalidade para idosos. ${ }^{24}$
A inatividade física contribui para a perda de massa muscular (falta de estímulos para a manutenção da massa e força muscular), portanto há a necessidade de estimular e incentivar os idosos a praticar atividade física, sobretudo exercícios anaeróbicos (exercícios de resistência) com papel preventivo e de recuperação para manutenção e/ou ganho de massa muscular e, consequentemente, de força muscular. ${ }^{4} \mathrm{O}$ sedentarismo pode contribuir para a perda funcional dos idosos, propiciada pela própria perda fisiológica de massa muscular e força, diminuindo a aptidão e desempenho físico, o que os torna ainda mais inativos. ${ }^{22}$

No presente estudo, não se observou diferença estatisticamente significativa nos valores do teste de FPP entre os idosos que praticavam exercício físico e entre os sedentários. É importante salientar, porém, que a prática de exercício físico foi autorreferida e não houve controle das informações relatadas, nem a separação do tipo de exercício em anaeróbico e aeróbico, o que prejudicou a análise dos dados.

\section{CONCLUSÕES}

Houve correlação estatisticamente significativa entre o estado nutricional classificado pela MiniAvaliação Nutricional e o teste de Força de Preensão Palmar na amostra total. Não houve diferença estatisticamente significativa entre $\mathrm{O}$ estado nutricional classificado pelo Índice de Massa Corpórea e o teste de FPP para homens e mulheres. Não foi observada diferença estatisticamente significativa entre o estado nutricional classificado pela circunferência da panturrilha (CP) e o teste de FPP. Assim, são necessários novos estudos, ampliando o tamanho da amostra, para que possam ser feitas inferências com base nos dados encontrados para a população em geral. 


\section{REFERÊNCIAS}

1. Giampaoli S, Ferrucci L, Cecchi F, Noce CLo, Poce A, Dima F, et al. Hand-grip strength predicts incident disability in non- disable older men. Age Ageing 1999; 28 (3): 283-88.

2. Alexandre TS, et al. Relação entre força de preensão manual e dificuldade no desempenho de atividades básicas de vida diária em idosos do município de São Paulo. Saúde Coletiva 2008 Ago; 5(24): 178-82.

3. Organização Pan-Americana da Saúde. Saúde, Bem-estar e Envelhecimento - O Projeto Sabe no município de São Paulo: uma abordagem inicial. Brasília: OPAS; 2003.

4. Silva TAA, Frisoli Junior A, Pinheiro MM, Szejnfeld VL . Sarcopenia Associada ao Envelhecimento: Aspectos Etiológicos e Opções Terapêuticas. Rev Bras Reumatol 2006; 46(6): 391-397.

5. Wallace JI, Schwartz RS. Epidemiology of weight loss in humans with special reference to wasting in the elderly. International Journal of cardiology 2002; 85:15-21.

6. Augusto ALP. Avaliação Nutricional. In: Augusto ALP, Alves DC, Mannarino IC, Gerudes M. Terapia Nutricional. São Paulo: Atheneu; 1995. 28-37.

7. Acuña K, Cruz T. Avaliação do Estado Nutricional de Adultos e Idosos e Situação Nutricional da População Brasileira. Arq Bras Endocrinol Metab 2004; 48(3).

8. Kuzu MA, Terzioglu H, Genç V, Erkek AB, Özban $\mathrm{M}$, Sonyürek P, et al. Preoperative nutritional risk assessment in predicting postoperative outcome in patients undergoing major surgery. World J Surg 2006; 30(3): 378-90.

9. Guigoz Y, Vellas B. A Mini avaliação nutricional (MAN) na classificação do estado nutricional do paciente idoso: apresentação, história e validação da MAN. In: Mini Avaliação Nutricional (MAN): pesquisa e prática no idoso. Nestlé Nutr Workshop Ser Clin Perform Programme 1998; 1: 01-02.

10. Gibson RS. Principles of Nutritional Assessment. New York: Oxford University Press 1990; 162-86.

11. Menezes TN, Marucci MFN. Perfil dos indicadores de gordura e massa muscular corporal dos idosos de Fortaleza, Ceará, Brasil. Cad Saúde Pública 2007; 23(12).
12. Bohannon RW. Hand-grip dynamometry provides a valid indication of upper extremity strength impairment in home care patients. J Hand Ther 1998; 11(4): 258-60.

13. AM Klidjian, KJ Foster, RM Kammerling, A Cooper, and SJ Karran. Relation of anthropometric and dynamometric variables to serious postoperative complications. BMJ 1980; 281(6245): 899-901.

14. Schlüssel MM, Anjos L, Kac G. A dinamometria manual e seu uso na avaliação nutricional. Rev Nutr 2008; 21(2): 223-235.

15. Rantanen T, Guralnik JM, Foley D, Masaki K, Leveille S, Curb JD, et al . Midlife Hand Grip Strength as a Predictor of Old Age Disability. JAMA 1999; 281(6): 558-560.

16. Pieterse S, Manandhar M, Ismail S. The association between nutritional status and handgrip strength in older Rwandan refugees. Eur.j.clin.nutr 2002; 56: 933-939.

17. Matsudo S, Araújo, T, Matsudo, V, Andrade, D, Andrade, E, Oliveira, LC, et al. Questionário Internacional de Atividade Física (I PAQ): Estudo de Validade e Reprodutibilidade no Brasil. Atividade Física\&Saúde 2001; 6(2).1-14

18. Desrosiers J, Bravo G, Hébert R, Dutil E. Normative data for grip strength of elderly man and woman. Am J Occup Ther 1995; 49: 637-644.

19. Hillman TE, Nunes QM, Hornby ST, Stanga Z, Neal KR, Rowlands BJ, Allison SP, et al. A practical posture for hand grip dynamometry in the clinical setting. Clin Nutr 2005; 24(2): 224-8.

20. Hohl Hohl CM, Dankoff J, Colacone A, Afilalo M. Polypharmacy adverse drug-related events, and potential adverse drug interactions in elderly patients presenting to an emergency department. Ann Emerg Med 2001; 38(6): 666-71.

21. Camarano AA, Kanso S, Mello JL. Como vive o idoso brasileiro? [texto na Internet]. In: Ipea; 2010 Mar 23; Brasília. [Acesso em 20 set 2010]. Disponível em: http://www.ipea.gov.br/sites/000/2/livros/ idososalem60/Arq_06_Cap_01.pdf

22. Moura PMLS. Estudo da força de preensão palmar em diferentes faixas etárias do desenvolvimento humano. Brasília. Tese [Mestrado em Ciências da Saúde] - Universidade de Brasília; 2008. 
23. Gale CR, Martyn CN, Cooper C, et al. Grip strength, body composition, and mortality. Int J Epidemiol 2007;36(1):228 -235.

24. Geraldes AAR, de Oliveira, ARM, Carvalho JM, FGarinatti PTV. A força de Preensão manual é Boa Preditora do Desempenho Funcional de idosos Frágeis: um Estudo Correlacional Múltiplo. Rev Bras Med Esporte 2008 Jan; 14(1): 11-16.
25. Oliveira FB. Força de preensão palmar em idosos institucionalizados do município de Goiânia, Goiás, Brasil: características gerais e relação com índice de Massa Corporal. Brasília. Tese [Mestrado em Ciências da Saúde] - Universidade de Brasília; 2009.

26. Garcia PA. Sarcopenia, mobilidade funcional e nível de atividade física em idosos ativos da comunidade. Belo Horizonte. Tese [Mestrado em Ciências da Reabilitação] - Universidade Federal de Minas Gerais; 2008.

Recebido: 20/9/2011

Revisado: 25/4/2012

Aprovado: 21/5/2012 